\title{
A novel curcumin derivative CL-6 exerts antitumor effect in human gastric cancer cells by inducing apoptosis through Hippo-YAP signaling pathway
}

This article was published in the following Dove Medical Press journal: OncoTargets and Therapy

Chenmin Ye, ${ }^{1,2, *}$ Wenqian Wang, ${ }^{3, *}$ Guojun Xia, ${ }^{1,2, *}$ Chengyang $\mathrm{Yu},{ }^{1,2}$ Yongdong $\mathrm{Yi}^{1}{ }^{1,2}$ Chunyan Hua, ${ }^{4}$ Fuyang Tu, ${ }^{1,2}$ Leibin Shen, ${ }^{1,2}$ Canjin Chen, ${ }^{1,2}$ Weijian Sun, ${ }^{\prime}$ Zhiqiang Zheng'

'Department of Gastrointestinal Surgery, The Second Affiliated Hospital and Yuying Children's Hospital of Wenzhou Medical University, Wenzhou, Zhejiang Province 325027, People's Republic of China; ${ }^{2}$ The Second School of Medicine, Wenzhou Medical University, Wenzhou, Zhejiang Province 325027, People's Republic of China; ${ }^{3}$ Department of Breast Surgery, The Second Affiliated Hospital and Yuying Children's Hospital of Wenzhou Medical University, Wenzhou, Zhejiang Province 325027, People's Republic of China; ${ }^{4}$ School of Basic Medical Sciences, Wenzhou Medical University, Wenzhou, Zhejiang Province 325035, People's Republic of China

*These authors contributed equally to this work

Correspondence: Weijian Sun; Zhiqiang Zheng

Department of Gastrointestinal Surgery, The Second Affiliated Hospital and Yuying Children's Hospital of Wenzhou Medical University, No. 109, Xueyuan West Road, Lucheng District, Wenzhou, Zhejiang Province 325027, People's Republic of China

Email fame198288@I26.com; zzq652992@163.com
Purpose: Gastric carcinoma is the second most frequently diagnosed cancer and leading cause of cancer death in China. As a new generation of cancer therapeutic drug, CL-6, a curcumin derivative, shows better bioavailability than curcumin, which has shown anticancer effects in gastric cancer (GC). However, whether CL-6 shows similar activities in GC has not been examined.

Materials and methods: Cell proliferation assay, colony-forming assay, flow cytometric analysis, wound healing assay, and Transwell invasion assay were performed to examine the effects of CL-6 on proliferation, apoptosis, migration, and invasion on human AGS and MGC-803 cell lines. Western blot was used to evaluate protein levels of Bax, Bcl-2, YAP, p-YAP, and Lats, and gene expression was measured by real-time quantitative PCR (RT-qPCR).

Results: CL-6 dose dependently reduced proliferation, increased apoptosis, and inhibited the migration and invasion abilities of AGS and MGC-803 cells. CL-6 also increased levels of pro-apoptotic protein Bax, decreased levels of antiapoptotic protein Bcl-2, and increased the $\mathrm{Bax} / \mathrm{Bcl}-2$ ratio. CL- 6 treatment also inhibited YAP and YAP protein and mRNA expression, while it induced the expression of Lats and p-YAP (Ser127).

Conclusion: CL-6 induces apoptosis of GC cells by activating the Hippo-YAP signaling pathway. These results indicate the therapeutic potential of the novel curcumin derivative CL-6 in GC. Keywords: gastric cancer, apoptosis, migration, YAP, Hippo-YAP pathway

\section{Introduction}

Gastric cancer (GC) is the fifth most common malignancy and the third leading cause of cancer-related deaths worldwide. In East Asia, the incidence of GC is significantly higher. ${ }^{1}$ In China, GC is the second most commonly diagnosed cancer; the number of new cases reported in 2015 was estimated at 679,100, accounting for more than $40 \%$ of the global annual incidence rate. ${ }^{2} \mathrm{GC}$ is a heterogeneous disease, not only at the pathological and clinical levels but also at the molecular level. ${ }^{3,4}$ In recent years, several systemic therapies have been developed for GC that have shown promise, but GC remains the leading cause of death in China. ${ }^{4,5}$ Therefore, the development of new drugs for GC treatment is critical.

Curcumin is a polyphenol and the active component of turmeric and ginger, which has had a long history of use in traditional medicine. Curcumin has shown multiple pharmacological functions such as hypolipidemic, antimutagenic, anticancer, and antioxidation activities, and thus, curcumin has been investigated as a therapeutic drug in multiple clinical studies in many diseases. ${ }^{6}$ Recent studies demonstrated that curcumin shows significant apoptosis-inducing abilities in breast cancer, lung cancer, pancreatic cancer, and GC in vitro and in vivo. ${ }^{7-10}$ Although curcumin has been 
examined in many clinical trials, its widespread applications are limited because of its poor bioavailability, obvious firstpass elimination, and easy hydrolysis of aqueous solution. ${ }^{11}$ Curcumin derivatives and analogues could increase bioavailability via structural modification. ${ }^{12}$ We previously synthesized a series of curcumin derivatives, one of which showed lead-like properties, which were more active than curcumin in the inhibition of GC. Here, we investigated a novel curcumin derivative (3E,5E)-3,5-bis(4-(chloro)benzylidene)4-piperidinone $\left(\mathrm{C}_{19} \mathrm{H}_{15} \mathrm{ONCl}_{2}\right.$; molecular weight: 343.0525; CL-6; Figure 1A) for its anticancer effects and the potential mechanism in human GC cells.

Here, using human AGS and MGC-803 cell lines, we assessed the effects of CL-6 on cell proliferation, invasion, migration, and apoptosis of GC cells in vitro and found that the CL- 6 exhibited novel antitumor effects in GC by inducing apoptosis. Finally, we proposed CL-6 as a strong therapeutic potential to activate the Hippo-YAP signaling pathway for the treatment of GC.

\section{Materials and methods}

\section{Synthesis and dissolution of CL-6}

CL-6 was designed and synthesized by Prof. Renshan Ge from Wenzhou Medical University, which was synthesized, identified, and dissolved in dimethyl sulfoxide (DMSO). CL-6 was dissolved in DMSO for experiments. The water used in the experiments was thrice distilled using a Milli-Q Biocel system (EMD Millipore, Billerica, MA, USA).

\section{Cell culture}

Human AGS and MGC-803 GC cell lines were purchased from the Cell Bank of Type Culture Collection of Chinese Academy of Sciences, Shanghai Institute of Cell Biology. AGS cells were cultured in the minimum essential medium (F-12K; Thermo Fisher Scientific, Waltham, MA, USA) supplemented with $10 \% \mathrm{FBS}$ at $37^{\circ} \mathrm{C}$ in a humidified atmosphere in the presence of $5 \% \mathrm{CO}_{2}$. MGC-803 cells were cultured in DMEM (Thermo Fisher Scientific) supplemented with $10 \% \mathrm{FBS}$ at $37^{\circ} \mathrm{C}$ in a humidified atmosphere in the presence of $5 \% \mathrm{CO}_{2}$.

\section{Cell proliferation assays}

Cell proliferation was evaluated using the Cell Counting Kit-8 (CCK-8) according to the manufacturer's instructions (Dojindo Laboratories, Kumamoto, Japan). AGS and MGC-803 cells $\left(7 \times 10^{3}\right)$ were seeded in a 96 -well plate overnight. Cells were then treated with different concentrations of CL- 6 for 24, 48, and 72 hours. The OD values were determined by a VersaMax microplate reader at $450 \mathrm{~nm}$.
The effect of CL-6 on cell viability was assessed by colorimetric immunoassay (Cell-Light ${ }^{\mathrm{TM}}$ EdU Apollo567 In Vitro Imaging Kit; Ribobio, Guangzhou, China) according to the manufacturer's instructions. Cells $\left(1 \times 10^{4}\right)$ were cultured in 24-well plates and then treated with various concentrations of CL-6 $(2.5,5.0$, and 7.5 $\mu \mathrm{M})$ or DMSO. The immunoassay was performed 48 hours after CL-6 incubation. The final concentration of DMSO was kept at $<0.05 \%$ in all wells. The experiments were repeated three times for each condition.

\section{Colony-forming assay}

AGS and MGC- 803 cells $\left(3 \times 10^{5}\right.$ cells per well) were plated in six-well plates and incubated overnight. After 48 hours exposure to different concentrations of CL- 6 , the viable cells were collected and counted. A total of 500 cells were collected, seeded in a $100 \mathrm{~mm}$ dish, and subsequently incubated for 14 days at $37^{\circ} \mathrm{C}$ in a humidified $5 \% \mathrm{CO}_{2}$ atmosphere. Colonies were stained with $2 \%$ crystal violet.

\section{Flow cytometric analysis}

Apoptosis was analyzed using the Annexin V-FITC Apoptosis Detection Kit (KeyGen Biotech, Nanjing, China) according to the manufacturer's protocol. After treatment with various concentrations of CL- 6 for 48 hours, cells were trypsinized, washed in cold PBS, and then harvested by centrifugation. The collected cells were resuspended in $500 \mu \mathrm{L}$ of binding buffer containing $5 \mu \mathrm{L}$ propidium iodide (PI) and $5 \mu \mathrm{L}$ Annexin V-FITC and then stained in the dark for 20 minutes at room temperature. Cells were analyzed by a Beckman Coulter CyotFLEX flow cytometer.

\section{Wound healing assay}

Cell were cultured in a six-well culture plate until they reached $90 \%$ confluence. A pipette tip was then used to create a scratch in the well in the longitudinal axis. Detached cells were carefully removed from the plates with PBS. Next, the medium containing $10 \%$ FBS was added, and the plate was incubated at $37^{\circ} \mathrm{C}$ in $5 \% \mathrm{CO}_{2}$ incubator. At 0,24 , and 48 hours, the plates were observed using an inverted phase contrast microscope and photographed. Experiments were repeated at least three times.

\section{Transwell invasion assay}

The invasive capacity of cancer cells was determined using a Transwell chamber $(8 \mu \mathrm{m}$ pore size; Corning Incorporated, Corning, NY, USA) with Matrigel (BD Biosciences, San Jose, CA, USA) according to the manufacturer's instructions. Briefly, cells $\left(1 \times 10^{5}\right.$ cells/well $)$ treated with CL-6 for 48 hours were placed into the upper chamber in $200 \mu \mathrm{L}$ 
A<smiles>O=C1/C(=C/c2ccc(Cl)cc2)CNC/C1=C\c1ccc(Cl)cc1</smiles>

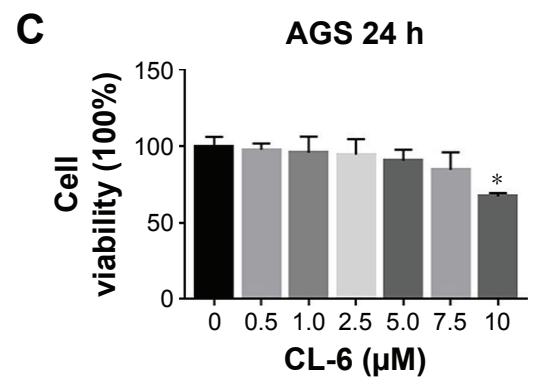

MGC-803 24 h

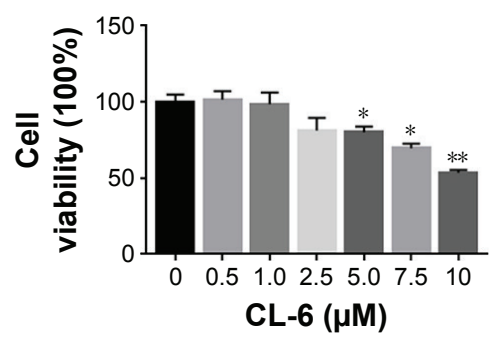

E

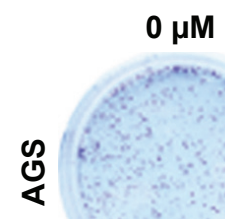

$2.5 \mu \mathrm{M}$

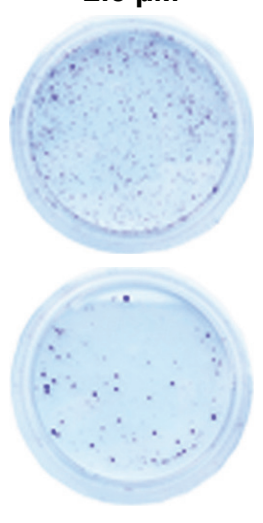

B

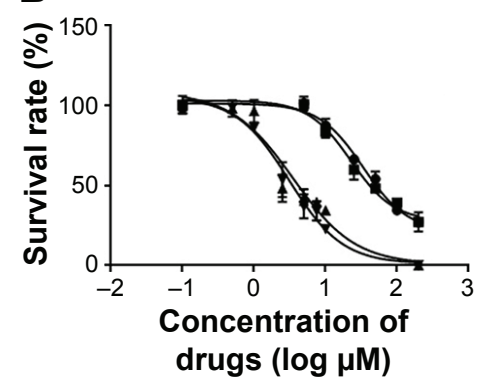

- AGS cur IC $I_{50}=59.52 \mu \mathrm{M}$

- MGC-803 cur $\mathrm{IC}_{50}=54.46 \mu \mathrm{M}$

- AGS CL- $6 \mathrm{IC}_{50}=4.32 \mu \mathrm{M}$

₹ MGC-803 CL-6 IC $50=3.57 \mu \mathrm{M}$

AGS $48 \mathrm{~h}$

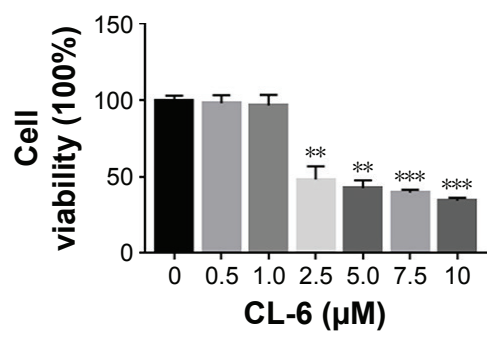

MGC-803 48 h

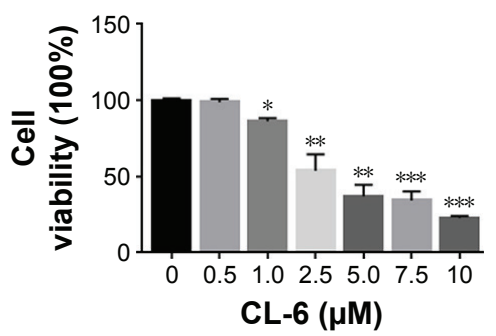

CL-6 $(\mu \mathrm{M})$

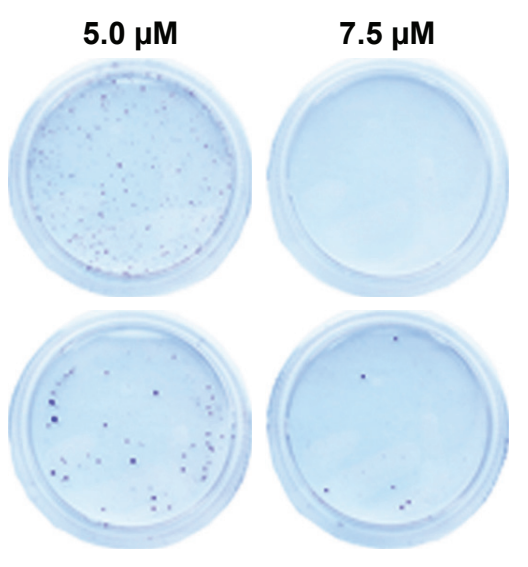

D

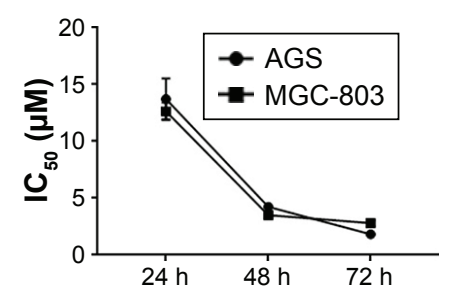

AGS $72 \mathrm{~h}$

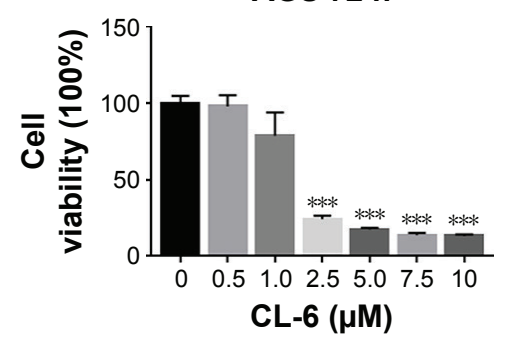

MGC-803 72 h

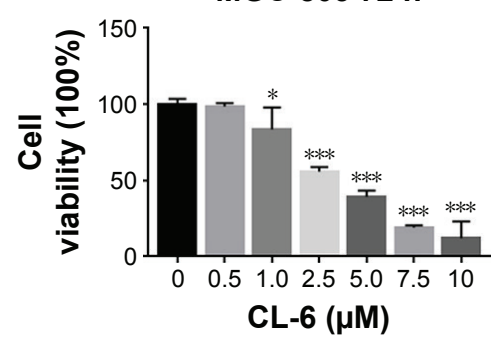

AGS
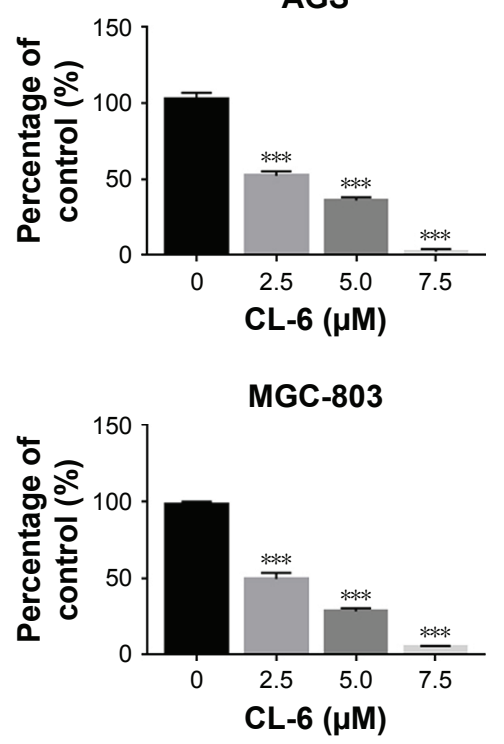

Figure I CL-6 inhibits the growth of GC cells.

Notes: (A) Molecular structure of the curcumin derivative CL-6. (B) Curcumin and the curcumin derivative CL-6 inhibit gastric cancer cell growth. Effect of curcumin and CL-6 treatment for 48 hours on GC cell growth as detected by the CCK-8 assay. (C) CCK-8 assays of GC cell growth after treatment with CL-6 for 24,48 and 72 hours. (D) Colony-forming assay was performed on GC cells treated with CL-6. (E) IC 50 of CL-6 after 24-, 48-, and 72-hour treatment in GC cells. Data were shown as mean \pm SD from three independent experiments. $* P<0.05$, $* * P<0.01$, and $* * * P<0.001$.

Abbreviations: GC, gastric cancer; CCK-8, Cell Counting Kit-8; IC ${ }_{50}$, half maximal inhibitory concentration. 
of serum-free DMEM. In the lower chamber, $500 \mu \mathrm{L}$ of complete medium (containing 10\% FBS) was added. After 24-hour incubation at $37^{\circ} \mathrm{C}$ with $5 \% \mathrm{CO}_{2}$, the medium was removed from the upper chamber. The noninvaded cells on the upper side of the chamber were scraped off with a cotton swab. Cells that migrated from the Matrigel into the pores of the inserted filter were fixed with $100 \%$ methanol, stained with hematoxylin, mounted, and dried at $80^{\circ} \mathrm{C}$ for 30 minutes. The numbers of cells invading through the Matrigel were counted in three randomly selected visual fields from the central and peripheral portions of the filter using an inverted microscope (200× magnification). Each assay was repeated three times.

\section{Western blot analysis}

Cells were harvested and lysed in cell-lysis buffer (Cell Signaling Technology, Danvers, MA, USA). Protein concentrations were quantified by a Bradford assay kit (BioRad Laboratories Inc., Hercules, CA, USA) according to the manufacturer's instructions. Samples were heated for 10 minutes at $100^{\circ} \mathrm{C}$, and then, equal amounts of denatured protein samples were separated by gel electrophoresis using $12 \%$ SDS-PAGE and transferred electrophoretically to PVDF membranes. Membranes were incubated at $4^{\circ} \mathrm{C}$ overnight with primary antibody $(1: 1,000)$ and washed three times with TBST buffer. The membranes were then incubated with the horseradish peroxidase-conjugated secondary antibody at room temperature for 2 hours. Protein bands were visualized using ECL reagent (Pierce, Rockford, IL, USA). Primary antibodies against YAP, p-YAP (Ser127), Bax, Bcl-2, Lats, and $\beta$-actin were purchased from Abcam (Cambridge, MA, USA).

\section{Real-time quantitative PCR (qPCR)}

Total RNA was extracted from cells using TRIzol reagent (Thermo Fisher Scientific) according to the manufacturer's instructions and then reverse transcribed into cDNA using PrimeScript RT Reagent (Takara). Real-time PCR was performed using a Real-time PCR System (Roche LightCycler 480) with SYBR Green qPCR Master Mix (Thermo Fisher Scientific) to measure the expression levels of Lats and YAP mRNA. GAPDH mRNA was used as endogenous control. Relative quantification of gene expression was repeated in three independent experiments. Primer sequences are as follows: YAP: sense: 5'-TTCAAGGTAGTCTGGGAAACG-3', antisense: 5'-TGAGAACAATGACGACCAATAG-3'; Lats: sense: 5'-TGTTTGGCACCATCATAGACT-3', antisense: 5'-TAACTTTCCATCAGGGAGACC-3'; and $\beta$-actin: sense: 5'-CTACCTCATGAAGATCCTCACCGA-3',

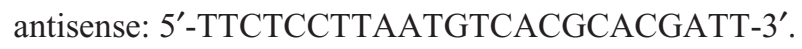

\section{Statistical analyses}

Data were analyzed using GraphPad Prism 7.0 (GraphPad Software, Inc., La Jolla, CA, USA) and SPSS v21.0 software (IBM Corporation, Armonk, NY, USA). Results are presented as mean $\pm \mathrm{SD}$. For all experiments, one-way ANOVA or Student's $t$-test was used to analyze the differences between groups. $P$-values were derived from two-sided tests, and $P<0.05$ was considered as statistically significant.

\section{Results \\ CL-6 inhibits viability, colony formation, and proliferation of GC cells}

We first compared the effects of CL- 6 and curcumin on GC cells and found that CL-6 inhibited the growth of GC cells more potently than curcumin (Figure 1B). To investigate the potential cytotoxicity of CL-6 on GC cells, we performed CCK-8 assay, colony formation assay, and cell proliferation assay in AGS and MGC-803 cells treated with different concentrations of CL- 6 for 24, 48, and 72 hours. CCK- 8 assays showed that CL-6 significantly reduced the viabilities of GC cells in dose- and time-dependent manners compared with control treatments (Figure 1C). The half maximal inhibitory concentration $\left(\mathrm{IC}_{50}\right)$ values of CL-6 for AGS cells were $13.72 \pm 1.80,4.23 \pm 0.05$, and $1.82 \pm 0.04 \mu \mathrm{M}$ after 24-, 48-, and 72-hour treatment, respectively. The $\mathrm{IC}_{50}$ values for MGC-803 cells were 12.64 $\pm 7.6,3.50 \pm 0.09$, and $2.85 \pm 0.26 \mu \mathrm{M}$ after 24-, 48-, and 72-h treatment, respectively (Figure 1D). Colony formation assays showed that CL-6 significantly reduced the colony formation of GC cells in a dose-dependent manner compared with control cells (Figure 1E). Furthermore, EdU analysis showed that CL-6 dose dependently reduced the proliferation of both AGS and MGC-803 cells (Figure 2). Together these results indicate that CL-6 exhibits effects in inhibiting the viability, colony formation, and proliferation of GC cells.

\section{CL-6 induces apoptosis in GC cells}

To clarify whether the growth inhibitory effects of CL-6 on AGS and MGC-803 cells involved the induction of apoptosis, we used Annexin V-FITC/PI double fluorescence staining and flow cytometry analysis. As shown in Figure 3A, CL-6 treatment induced cell apoptosis in AGS and MGC-803 cells. The apoptosis rates of AGS cells were increased to $47.83 \pm 4.43,64.01 \pm 2.26$, and $77.90 \% \pm 5.44 \%$ of the control upon treatment of $2.5,5.0$, and $7.5 \mu \mathrm{M} \mathrm{CL}-6$, respectively. 

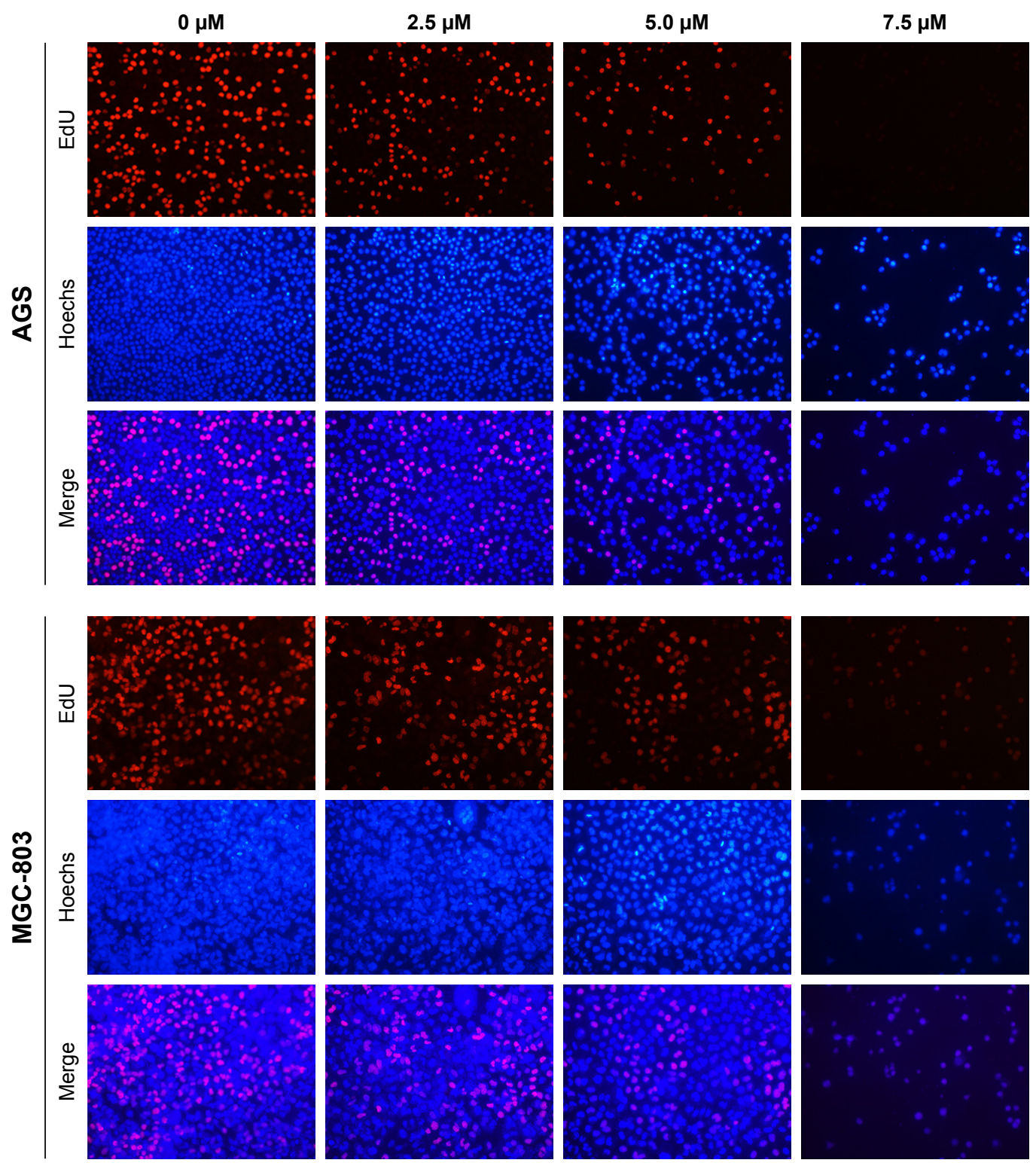

Figure 2 EdU assay in AGS and MGC-803 cells after 48-hour incubation with CL-6 (0, 2.5, 5.0, and 7.5 $\mu$ M) or DMSO as control.

Abbreviation: DMSO, dimethyl sulfoxide.

We observed similar apoptosis rates in MGC-803 cells (Figure $3 \mathrm{~A}$ and $\mathrm{B}$ ) We also examined the expressions of apoptotic markers Bcl-2 and Bax by Western blot and found that CL-6 increased the proapoptotic protein Bax, decreased the antiapoptotic protein $\mathrm{Bcl}-2$, and increased the Bax/Bcl-2 ratio (Figure 3C-E). These results showed that CL-6 induced apoptosis of GC cells.

\section{CL-6 inhibits migration and invasion of GC cells}

Metastasis is one of the main causes of cancer-related death. Therefore, we next examined the effects of CL- 6 on the migration and invasion of AGS and MGC-803 cells using wound-healing assay and Transwell assay. In wound-healing assays, we treated AGS and MGC-803 cells with different concentrations of CL-6 $(2.5,5.0$, and $7.5 \mu \mathrm{M})$ for 24,48 , and 72 hours, and we found that that CL- 6 significantly inhibited the migration of cancer cells in a dose-dependent manner (Figure 4A and B). Transwell assays showed that the number of invasive cells was significantly reduced at 48 hours after CL- 6 treatment; the number of invasive AGS cells was reduced to $51.69 \% \pm 4.10 \%$ and MGC- 803 cells were decreased to $46.17 \% \pm 7.14 \%$ in response to $2.5 \mu \mathrm{M}$ CL-6 compared with that of controls (Figure 4C and D) These results indicate that CL-6 inhibits the migration and invasion of GC cells. 

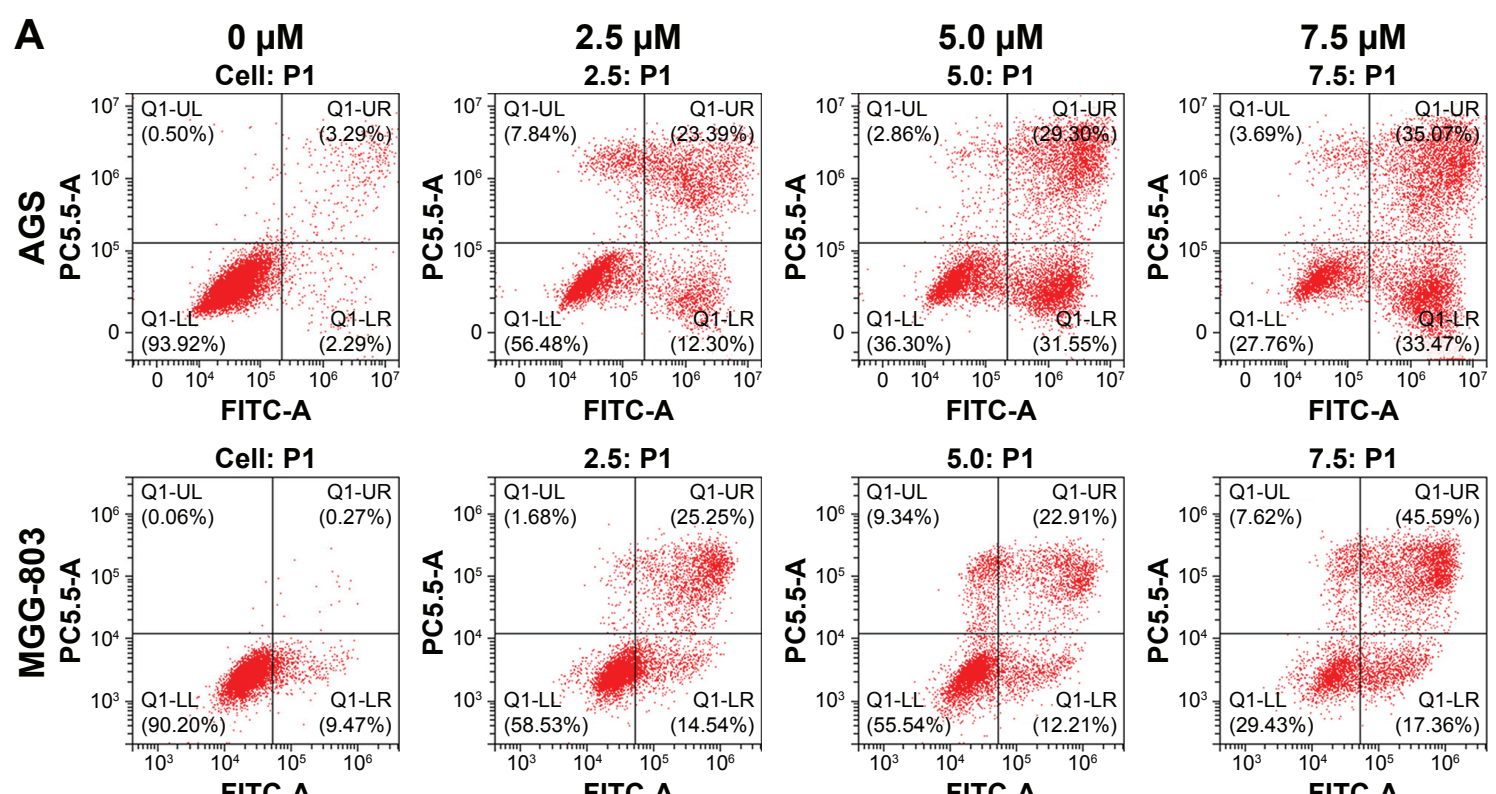

B
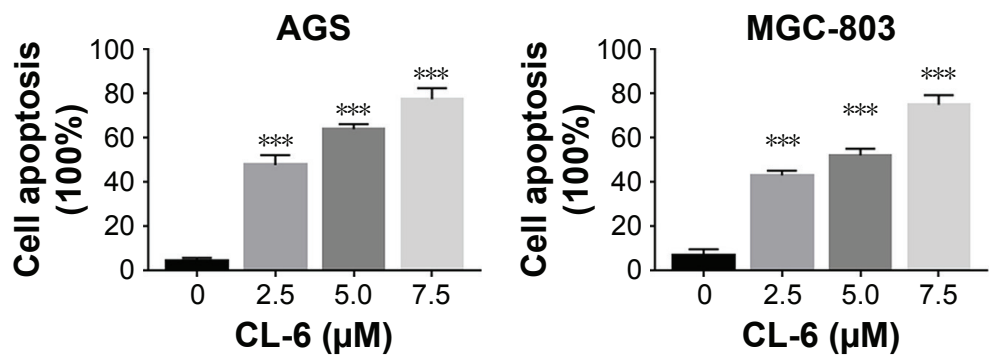

C
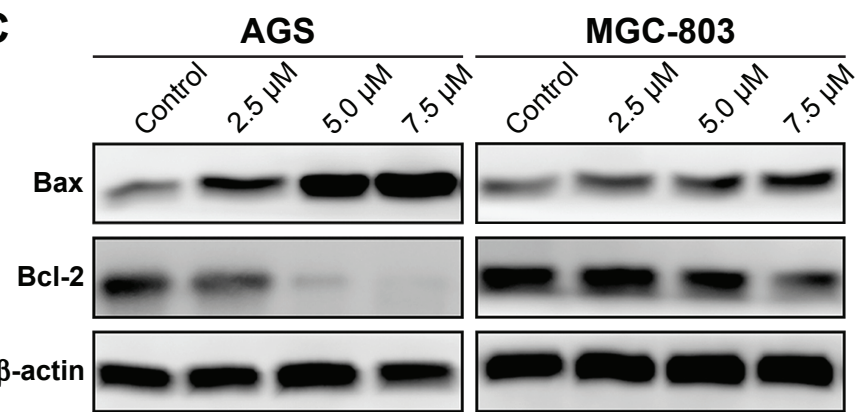

E
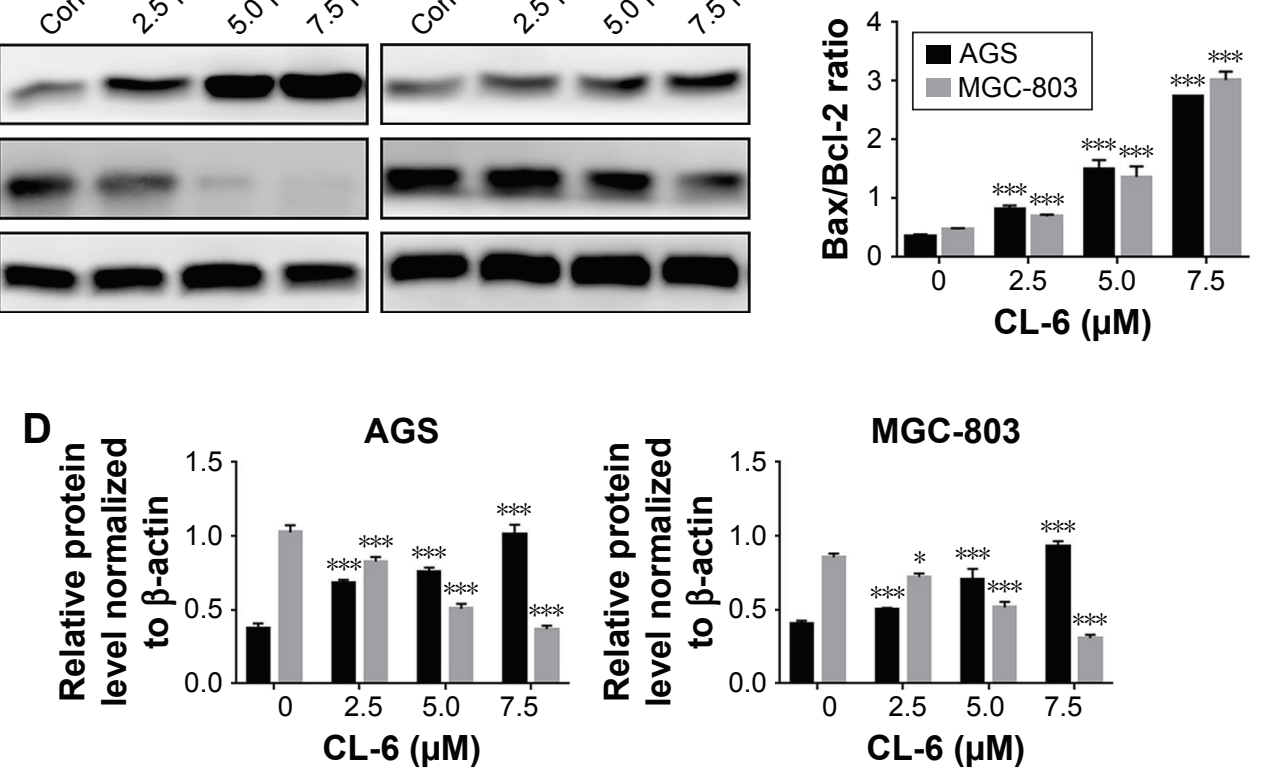

- Bax $\mathrm{Bcl}-2$

Figure 3 The effects of CL-6 on the apoptosis of GC cells.

Notes: (A) AGS and MGC-803 cells were treated with CL-6 (0, 2.5, 5.0 and $7.5 \mu \mathrm{M})$ for the indicated times. FITC and Annexin V/PI staining and flow cytometry were performed to evaluate apoptosis; (B) Quantification of results from (A). (C) AGS and MGC-803 cells were treated with CL-6 (0, 2.5, 5.0 and $7.5 \mu \mathrm{M})$ for 48 hours. Western blot was performed for phosphorylated Bax and Bcl-2. (D) Quantification of results from (C). (E) Ratio of Bax to $\mathrm{Bcl}-2$. $* P<0.05$ and $* * * P<0.00 \mathrm{l}$.

Abbreviations: GC, gastric cancer; PI, propidium iodide. 

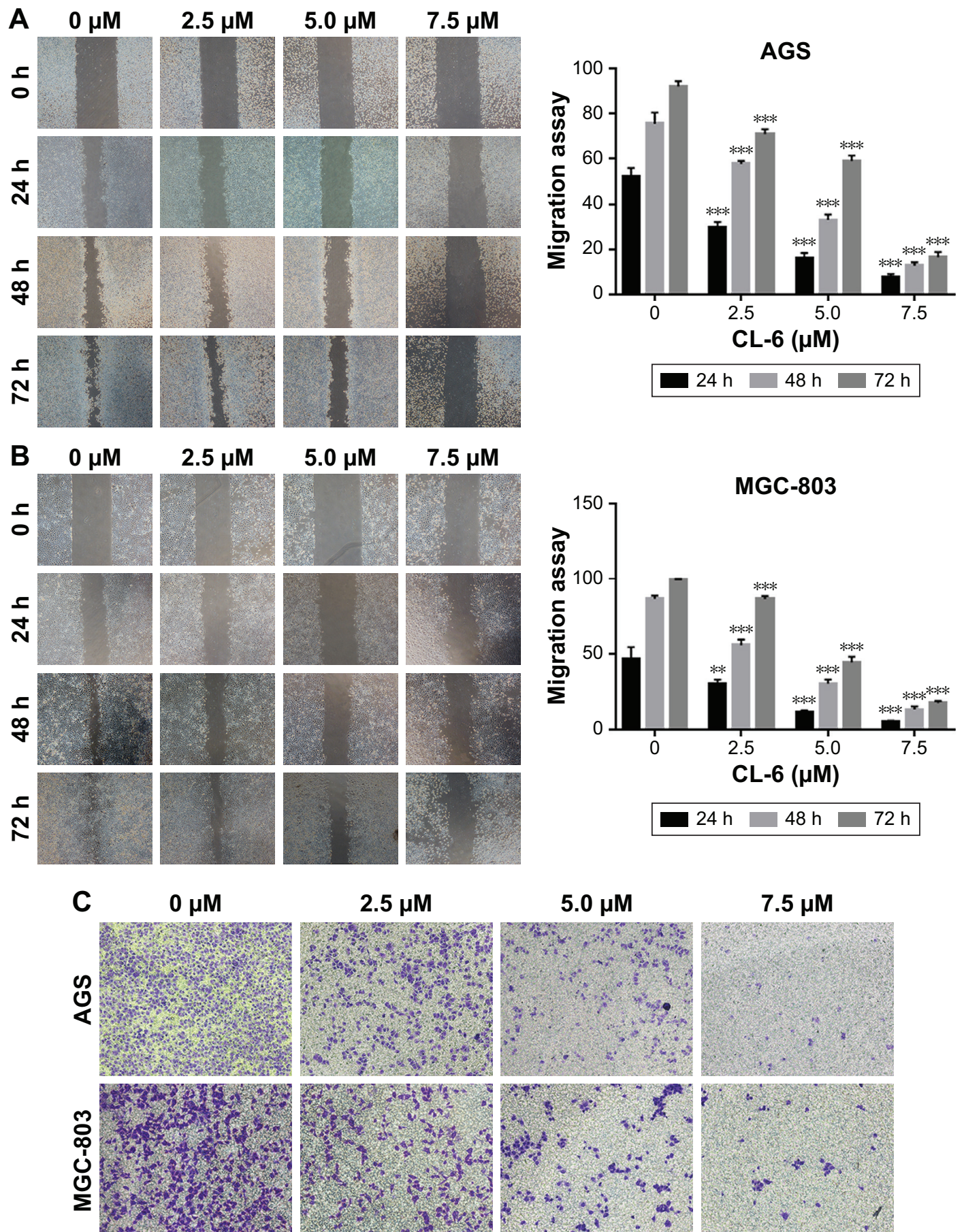

$2.5 \mu \mathrm{M}$

$5.0 \mu \mathrm{M}$

$7.5 \mu \mathrm{M}$
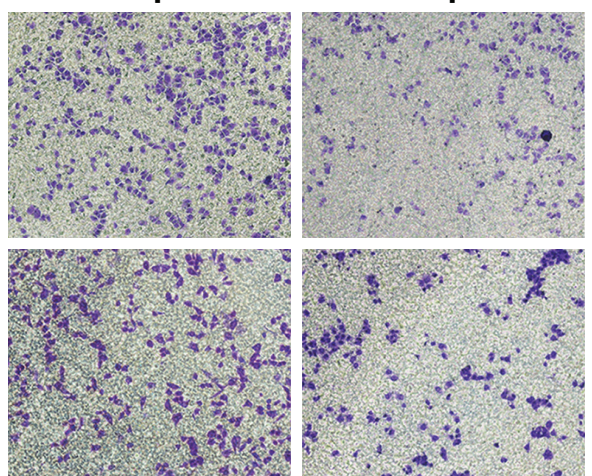

D
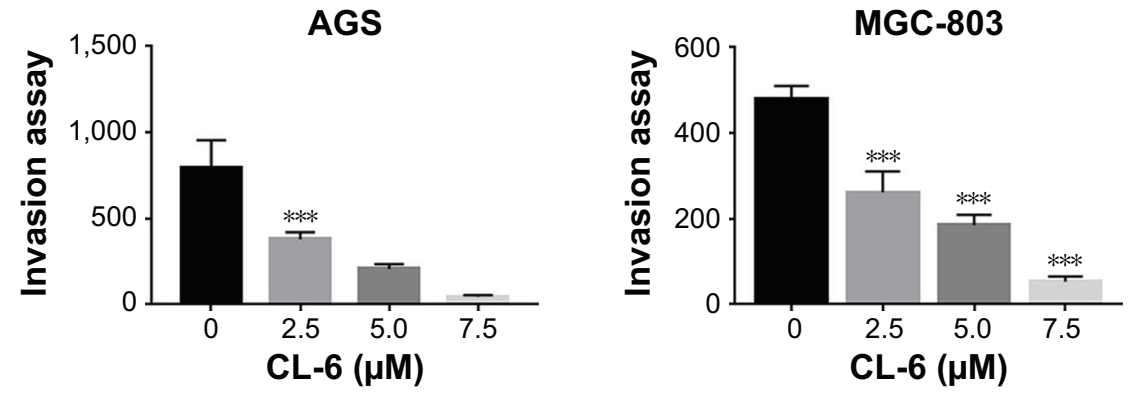

Figure 4 CL-6 inhibits the migration and invasion of GC cells.

Notes: (A) Wound healing assays were performed in AGS and MGC-803 cells treated with CL-6. (B) Quantification of results from (A). (C) Transwell invasion assays were performed in AGS cells and MGC-803 cells treated with CL-6. (D) Quantification of results from (C). ${ }^{* * P}<0.01$ and $* * * P<0.00 \mathrm{I}$.

Abbreviation: GC, gastric cancer. 


\section{CL-6 exerts its anticancer effect through the Hippo-Yap pathway}

In our previous study, we found that curcumin is an inhibitor of YAP expression. Thus, we examined whether CL-6 impacted YAP expression levels in GC cells. Western blot revealed that the expression of YAP was significantly decreased in GC cells treated with CL-6 compared with that in controls (Figure 5A and B). qPCR results also showed that CL-6 dose-dependently inhibited mRNA levels of YAP in GC cells (Figure 5E). Previous studies reported that the Hippo signaling pathway is the major regulator of YAP activity. Activation of Lats, the upstream member of the Hippo pathway, results in phosphorylation of its downstream molecule, YAP, which undergoes nuclear translocation and subsequent inactivation. ${ }^{13}$ Therefore, we examined the levels of Lats and p-YAP (Ser 127) in GC cells treated with CL-6. We found that CL-6 significantly induced the levels of Lats and p-YAP (Ser 127) in both cell lines compared with control treatment (Figure 5C and D). qPCR experiments found that CL-6 induced Lats mRNA expression in a dose-dependent manner, which is consistent with the Western blot results (Figure 5F). Our results suggest that CL-6 may exert its anticancer effects through the Hippo-YAP pathway.

\section{Discussion}

GC is the fifth most common cancer and the third leading cause of cancer-related deaths wordwide. ${ }^{1}$ The current treatment strategies for GC mainly include surgery, chemotherapy, radiotherapy, and molecular targeted therapy. Although new drugs and technologies have improved the therapeutic effects, the 5-year survival rate of GC patients is still $<24 \%{ }^{14}$ Curcumin was first proposed for the treatment of cancer in $1985,{ }^{15}$ and multiple studies have subsequently demonstrated that curcumin has anti-infective, anti-inflammatory, antioxidant, and antitumor growth effects. ${ }^{16-18}$ Owing to its anticancer spectrum, high efficiency, low toxicity, and no significant side effects, curcumin has been considered a third-generation anticancer drug and has caused widespread concern. ${ }^{16}$

Despite the demonstrated anticancer activities of curcumin, curcumin has poor stability in vitro, low bioavailability in vivo, and weak pharmacokinetic properties and thus still shows limitations regarding its use in clinical treatment. ${ }^{11}$ Researchers have shown that the curcumin derivatives and analogues that are generated through structural modifications are good strategies to improve the properties of curcumin. ${ }^{12}$ We previously synthesized a series of curcumin derivatives and found that CL-6 exhibited high absorption and bioavailability. Our preliminary study showed that the toxic effects of CL-6 on human GC cell lines were better than those of curcumin at the same concentration. Here, we demonstrated that CL-6 significantly inhibited the proliferation, invasion, and migration of GC cells in vitro and that CL-6 induced apoptosis in GC cells. Although curcumin has been investigated in a large number of clinical studies, the therapeutic effects and pharmacokinetic properties of CL-6 as a new curcumin derivative need further exploration. In preliminary experiments, CL-6 was not detectable by HPLC, because of the low sensitivity of our method. The development of a high-sensitivity detection method for CL-6 is required to enable future assays for concentrations of this compound throughout treatment.

Multiple studies have underscored the importance of YAP in the tumorigenesis of gastrointestinal cancers, and overexpression of YAP promotes the occurrence and progression of gastrointestinal tumors. ${ }^{19,20}$ Lentiviral vector-mediated silencing of YAP by siRNA inhibited the growth of GC and induced apoptosis of GC cells. ${ }^{19}$ Silencing of the YAP gene by RNA interference inhibited the invasion and metastasis of human SGC-7901 GC cells. ${ }^{21}$ Kang et al ${ }^{22}$ also found that YAP exhibited carcinogenic properties in GC and might be a prognostic biomarker and potential therapeutic target for GC. These studies suggest that YAP is a key transcriptional regulator of $\mathrm{GC}$ and might be a new target for the treatment of GC. We previously found that curcumin was a potent inhibitor of YAP expression. Therefore, we measured the expression of YAP in AGS and MGC-803 cells treated with different concentrations of CL- 6 and found that CL- 6 inhibited YAP expression in GC cell lines.

YAP is a major downstream factor in the Hippo pathway, which plays an important role in cell proliferation, differentiation, and DNA damage. ${ }^{23-25}$ Mutations in components of this pathway have been associated with various cancers. ${ }^{26-28}$ Recent studies have found that Ser127 phosphorylation of YAP is a key event in the translocation of YAP between the nucleus and cytoplasm. YAP phosphorylated at Ser127 binds cytoplasmic chaperones and remains in the cytoplasm. When Ser127 phosphorylation of YAP is reduced, YAP is released by its cytoplasmic chaperone and translocates into the nucleus, allowing it to modulate the transcription of target genes associated with cell growth. ${ }^{25}$ Our study found that the level of phosphorylated YAP (Ser127) protein was increased in the two GC cells lines treated with CL-6, while the total YAP level decreased and the level of Lats increased. These findings suggest that the anticancer effects of CL- 6 might be associated with the activation of the Hippo-YAP signaling 
A

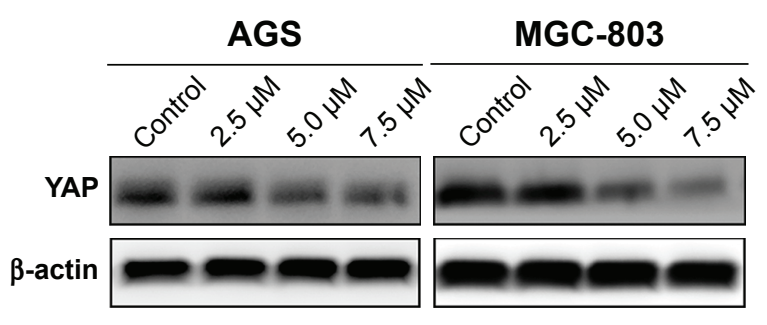

B

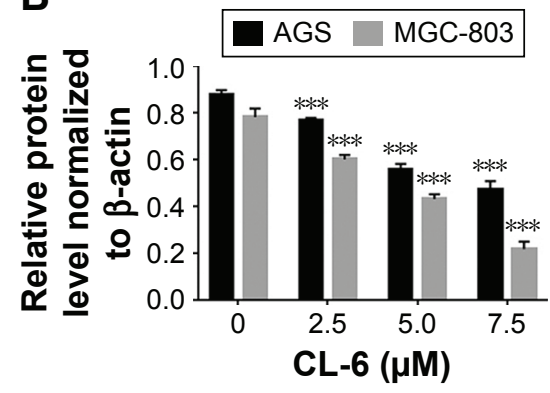

C

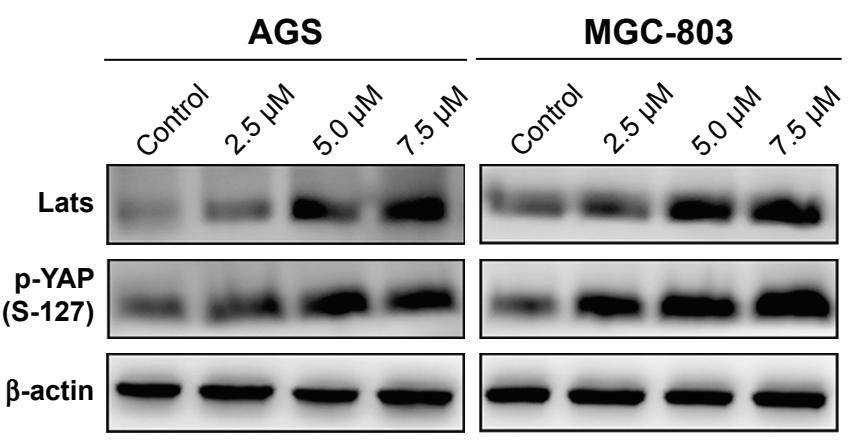

D
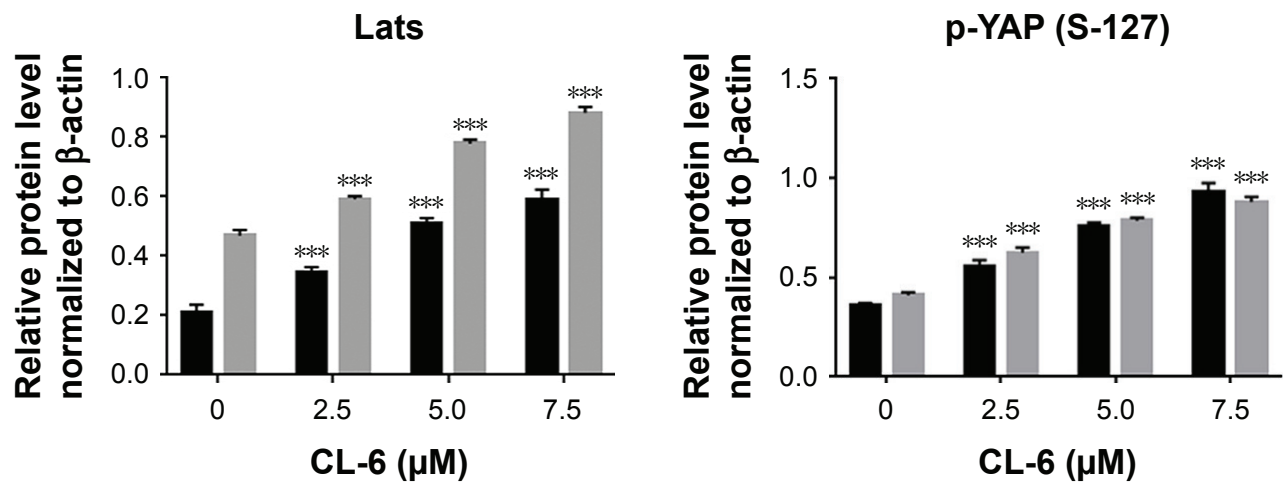

AGS MGC-803

E

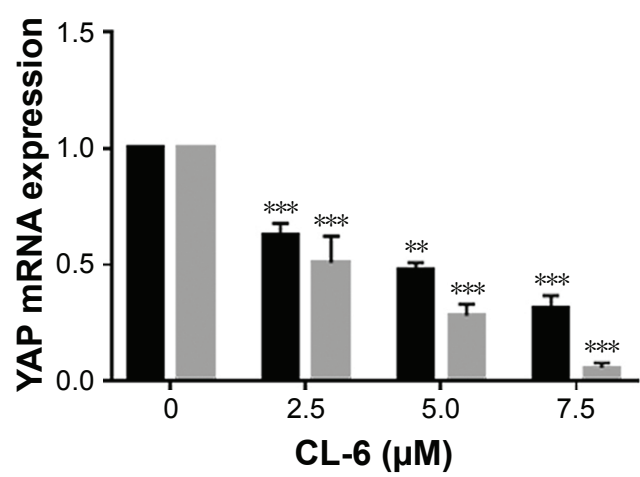

F

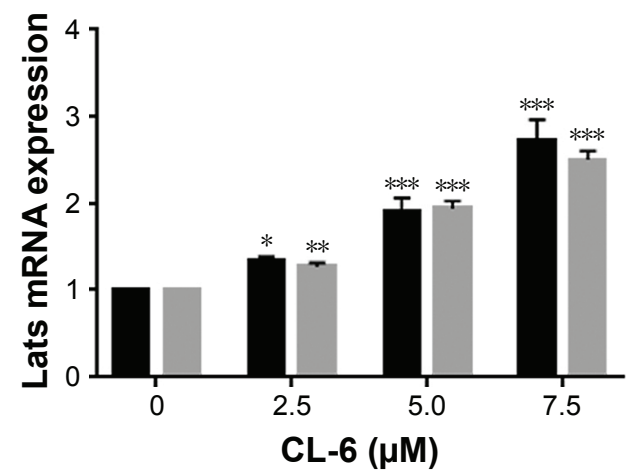

AGS MGC-803

Figure 5 CL-6 exhibits its anticancer effects through the Hippo-YAP pathway.

Notes: (A) Western blot analysis of YAP in cells treated with CL-6. (B) Quantification of results from (A). (C) Western blot analysis of Lats and P-YAP (Ser I 27) in cells. (D) Quantification of results from (C). (E and F) RT-qPCR of YAP and Lats mRNA in AGS and MGC-803 cells treated with CL-6 (0, 2.5, 5.0, and 7.5 $\mu$ M) for 48 hours. Data are represented as mean \pm SD from three independent experiments. $* P<0.05, * * P<0.01$, and $* * * P<0.00 \mathrm{I}$.

Abbreviation: RT-qPCR, real-time quantitative PCR. 
pathway. Future investigation of the detailed mechanisms of CL-6 in GC required.

In vitro studies showed that CL-6 exhibited anticancer effects in human GC cells. However, the functions of CL-6 in in vivo and clinical settings remain unclear. Recent studies have shown that curcumin exhibits significant apoptosisinducing abilities in animal models and breast cancer, lung cancer, pancreatic cancer, and GC in vitro. ${ }^{7-10}$ Some animal studies have shown that GC cell lines with curcumin or its analogues show a decrease in the volume, weight, and incidence of tumor. ${ }^{29,30}$ Although the characteristics of CL-6, as a curcumin derivative, are similar to curcumin, the precise function of CL-6 should be examined in future in vivo and clinical studies.

\section{Conclusion}

Here, we demonstrated the therapeutic potential of the novel curcumin derivative CL-6 in vitro. CL-6 inhibits the proliferation, migration, and invasion activities of GC cells. In addition, we found that CL-6 exhibits apoptosis-inducing activities in GC cells, and activation of the Hippo-YAP signaling pathway may be involved in these effects. Thus, we propose that the novel curcumin derivative CL-6 may be a useful candidate for the treatment of GC.

\section{Acknowledgments}

This work is supported by the Science and Technology Planning Project of Wenzhou City (no. Y20160050), Zhejiang Provincial Natural Science Foundation of China (no. LY18H160046), Zhejiang Medical Science Foundation (no. 2018KY532), and Medical and Health Science and Technology Plan Project of Zhejiang Province (no. 2018KY531).

\section{Disclosure}

The authors report no conflicts of interest in this work.

\section{References}

1. Bray F, Ferlay J, Soerjomataram I, Siegel RL, Torre LA, Jemal A. Global cancer statistics 2018: GLOBOCAN estimates of incidence and mortality worldwide for 36 cancers in 185 countries. CA Cancer J Clin. 2018. doi: $10.3322 /$ caac. 21492

2. Chen $\mathrm{W}$, Zheng R, Baade PD, et al. Cancer statistics in China, 2015. CA Cancer J Clin. 2016;66(2):115-132. doi:10.3322/caac.21338

3. Allemani C, Weir HK, Carreira H, et al. Global surveillance of cancer survival 1995-2009: analysis of individual data for 25676887 patients from 279 population-based registries in 67 countries (CONCORD-2). The Lancet. 2015;385(9972):977-1010. doi:10.1016/S01406736(14)62038-9

4. Strong VE, Wu AW, Selby LV, et al. Differences in gastric cancer survival between the U.S. and China. J Surg Oncol. 2015;112(1):31-37. doi: $10.1002 /$ jso. 23940
5. Lei Z, Tan IB, Das K, et al. Identification of molecular subtypes of gastric cancer with different responses to PI3-kinase inhibitors and 5-fluorouracil. Gastroenterology. 2013;145(3):554-565. doi:10.1053/j. gastro.2013.05.010

6. Ara SA, Mudda JA, Lingappa A, Rao P. Research on curcumin: a metaanalysis of potentially malignant disorders. J Cancer Res Ther. 2016; 12(1):175-181. doi:10.4103/0973-1482.171370

7. Bimonte S, Barbieri A, Palma G, et al. Dissecting the role of curcumin in tumour growth and angiogenesis in mouse model of human breast cancer. Biomed Res Int. 2015;2015:878134. doi:10.1155/2015/878134

8. Wang A, Wang J, Zhang S, Zhang H, Xu Z, Li X. Curcumin inhibits the development of non-small cell lung cancer by inhibiting autophagy and apoptosis. Exp Ther Med. 2017;14(5):5075-5080. doi:10.3892/ etm. 2017.5172

9. Su J, Zhou X, Wang L, et al. Curcumin inhibits cell growth and invasion and induces apoptosis through down-regulation of Skp2 in pancreatic cancer cells. Am J Cancer Res. 2016;6(9):1949.

10. Dhivya R, Ranjani J, Bowen PK, Rajendhran J, Mayandi J, Annaraj J. Biocompatible curcumin loaded PMMA-PEG/ZnO nanocomposite induce apoptosis and cytotoxicity in human gastric cancer cells. Mater Sci Eng. 2017;80:59-68. doi:10.1016/j.msec.2017.05.128

11. Anand P, Kunnumakkara AB, Newman RA, Aggarwal BB. Bioavailability of curcumin: problems and promises. Mol Pharm. 2007;4(6): 807-818. doi:10.1021/mp700113r

12. Mosley CA, Liotta DC, Snyder JP. Highly active anticancer curcumin analogues. Adv Exp Med Biol. 2007;595:77-103. doi:10.1007/978-0387-46401-5_2

13. Zhao B, Wei X, Li W, et al. Inactivation of YAP oncoprotein by the Hippo pathway is involved in cell contact inhibition and tissue growth control. Genes Dev. 2007;21(21):2747-2761. doi:10.1101/gad.1602907

14. Cui D, Zhang C, Liu B, et al. Regression of gastric cancer by systemic injection of RNA nanoparticles carrying both ligand and siRNA. Sci Rep. 2015;5:10726. doi:10.1038/srep10726

15. Kuttan R, Bhanumathy P, Nirmala K, George MC. Potential anticancer activity of turmeric (Curcuma longa). Cancer Lett. 1985;29(2):197-202.

16. Dai XZ, Yin HT, Sun LF, et al. Potential therapeutic efficacy of curcumin in liver cancer. Asian Pac J Cancer Prev. 2013;14(6):3855-3859.

17. Shishodia S, Chaturvedi MM, Aggarwal BB. Role of curcumin in cancer therapy. Curr Probl Cancer. 2007;31(4):243-305. doi:10.1016/j. currproblcancer.2007.04.001

18. Gupta SC, Patchva S, Aggarwal BB. Therapeutic roles of curcumin: lessons learned from clinical trials. AAPS J. 2013;15(1):195-218. doi:10.1208/s12248-012-9432-8

19. Lam-Himlin DM, Daniels JA, Gayyed MF, et al. The hippo pathway in human upper gastrointestinal dysplasia and carcinoma: a novel oncogenic pathway. Int J Gastrointest Cancer. 2006;37(4):103-109. doi:10.1007/s12029-007-0010-8

20. Da C-L. Significance and relationship between Yes-associated protein and survivin expression in gastric carcinoma and precancerous lesions. World J Gastroenterol. 2009;15(32):4055.

21. Zhou Z, Zhu JS, Xu ZP. RNA interference mediated YAP gene silencing inhibits invasion and metastasis of human gastric cancer cell line SGC7901. Hepatogastroenterology. 2011;58(112):2156-2161. doi:10.5754/ hge 11234

22. Kang W, Tong JH, Chan AW, et al. Yes-associated protein 1 exhibits oncogenic property in gastric cancer and its nuclear accumulation associates with poor prognosis. Clin Cancer Res. 2011;17(8):2130-2139. doi:10.1158/1078-0432.CCR-10-2467

23. Cottini F, Hideshima T, Xu C, et al. Rescue of Hippo coactivator YAP1 triggers DNA damage-induced apoptosis in hematological cancers. Nat Med. 2014;20(6):599-606. doi:10.1038/nm.3562

24. Keshet R, Adler J, Ricardo Lax I, et al. c-Abl antagonizes the YAP oncogenic function. Cell Death Differ. 2015;22(6):935-945. doi:10.1038/ cdd.2014.182

25. Moon S, Kim W, Kim S, et al. Phosphorylation by NLK inhibits YAP-14-3-3-interactions and induces its nuclear localization. EMBO Rep. 2017;18(1):61-71. doi:10.15252/embr.201642683 
26. Harvey K, Tapon N. The Salvador-Warts-Hippo pathway - an emerging tumour-suppressor network. Nat Rev Cancer. 2007;7(3):182-191. doi: $10.1038 / \mathrm{nrc} 2070$

27. Pan D. The hippo signaling pathway in development and cancer. Dev Cell. 2010;19(4):491-505. doi:10.1016/j.devcel.2010.09.011

28. Chan SW, Lim CJ, Chen L, et al. The Hippo pathway in biological control and cancer development. J Cell Physiol. 2011;226(4):928-939. doi:10.1002/jcp. 22435
29. Zou P, Zhang J, Xia Y, et al. ROS generation mediates the anti-cancer effects of WZ35 via activating JNK and ER stress apoptotic pathways in gastric cancer. Oncotarget. 2015;6(8):5860-5876. doi:10.18632 oncotarget.3333

30. Liu X, Sun K, Song A, Zhang X, Zhang X, He X. Curcumin inhibits proliferation of gastric cancer cells by impairing ATP-sensitive potassium channel opening. World J Surg Oncol. 2014;12:389. doi:10.1186/ 1477-7819-12-389

\section{Publish your work in this journal}

OncoTargets and Therapy is an international, peer-reviewed, open access journal focusing on the pathological basis of all cancers, potential targets for therapy and treatment protocols employed to improve the management of cancer patients. The journal also focuses on the impact of management programs and new therapeutic agents and protocols on

\section{Dovepress}

patient perspectives such as quality of life, adherence and satisfaction. The manuscript management system is completely online and includes a very quick and fair peer-review system, which is all easy to use. Visit http://www.dovepress.com/testimonials.php to read real quotes from published authors.

Submit your manuscript here: http://www.dovepress.com/oncotargets-and-therapy-journal 